\title{
Transfers of care between healthcare professionals in obstetric units of different sizes: the MidconBirth study
}

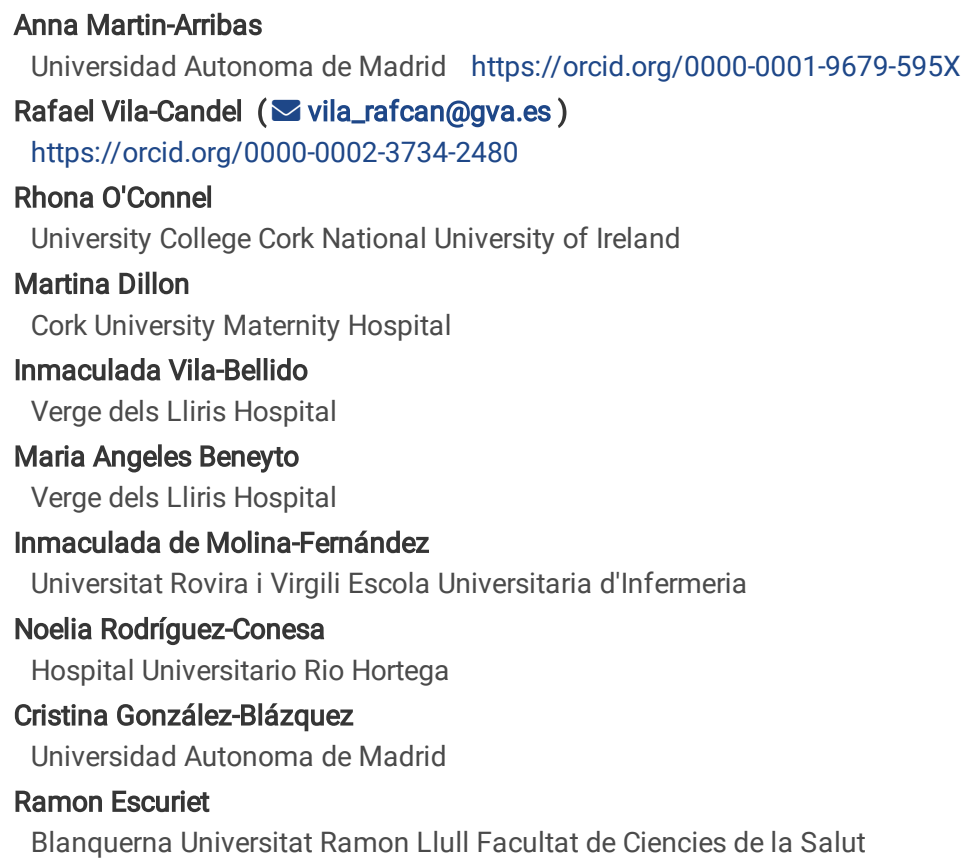

Research article

Keywords: Midwife-led care, obstetrician-led care, continuity of care, transfer of care, maternal outcomes, neonatal outcomes

Posted Date: December 9th, 2019

DOI: https://doi.org/10.21203/rs.2.18519/v1

License: (c) (1) This work is licensed under a Creative Commons Attribution 4.0 International License. Read Full License 


\section{Abstract}

Background: In Europe, the majority of healthy women give birth at conventional obstetric units with the assistance of registered midwives. Childbirth assistance offered in highly technological settings and the variability found in the intrapartum interventions performed are causes for concern. This study examines the relationships between the intrapartum transfer of care from midwife to obstetrician-led maternity care, obstetric unit size (OUS) with different degrees of midwifery autonomy, intrapartum interventions and birth outcomes.

Methods: A prospective, multicenter, cross-sectional study promoted by the COST Action IS1405 was carried out at eight public hospitals in Europe. Data were collected through an online platform in 2016-2019. The primary outcome was transfer of care (TOC). The secondary outcomes included type of onset of labor, oxytocin stimulation, epidural analgesia, type of birth, episiotomy/perineal injury, postpartum hemorrhage, early initiation of breastfeeding and early skin-to-skin contact. Descriptive statistics were used to summarize the women's characteristics. The Chi-square test was used to analyze the statistical significance of the differences in the percentages of hospital groups between the variable categories; an odds ratio (OR) with a $95 \% \mathrm{Cl}$, was calculated. $\mathrm{A}$ logistic regression was performed to ascertain the effects of studied co-variables on the likelihood that participants had a transfer of care. The significance level was set at $\mathrm{p}<0.05$.

Results: Out of a total of 2,126 low-risk women, those whose intrapartum care was initiated by a midwife $(1,772)$ were selected. There were statistically significant differences between transfer of care and OUS ( $1=29.0 \%, S 2=44.0 \%, S 3=52.9 \%, S 4=30.2 \%, p<0.001)$. Statistically significant differences between OUS and onset of labor, oxytocin stimulation, type of birth and episiotomy or perineal injury were observed $(p=0.009, p<0.001, p<0.001, p<0.001$ respectively).

Conclusion: Findings suggest that the model of care and OUS have a significant effect on the prevalence of intrapartum transfer of care and the birth outcomes. Future research should examine how models of care differ as a function of the OUS in a hospital, as well as the cost-effectiveness for the health care system. Keywords: Midwife-led care; obstetrician-led care; continuity of care; transfer of care, maternal outcomes, neonatal outcomes

\section{Background}

The past few years have witnessed the emergence of a worldwide debate about the care that healthy women receive when they are giving birth. This debate includes differing opinions as to the use of various technologies for normal or low-risk procedures [1], the concept of labor as a physiological process that is not to be understood in solely medical terms, and the growing role women themselves are taking in the decision-making processes affecting their care [2]. Additionally, the increase in the frequency of interventions carried out during labor, especially of cesarean sections, is a source of concern for a number of organization and for health care officials.

The organizational structure of maternity services might have an influence on the health outcomes of women and newborns [3]. The way these medical services are organized determines the level of continuity of care offered and the choice of which professionals provide what kinds of care throughout the process of pregnancy, labor and postpartum care. A number of studies have shown positive results when midwives are the primary providers of care throughout the process [4].

Maternity care in Europe is offered by organizations with a number of different kinds of structures, settings and locations. The degree to which European health care systems offer coverage varies from country to country. In some places, women are guaranteed coverage for the whole range options, whether women choose home births, birth centers, maternity units attended by midwives or conventional obstetric units. The size of maternity units also vary as does the degree of midwifery autonomy. The most common model in European countries is that of providing care in conventional obstetrics units. This translates into a situation in which most healthy women receive care in highly technological settings. Additionally, the frequency of intrapartum interventions varies greatly from place to place.

The quality of care provided to women during labor has been extensively studied by a number of researchers. Nonetheless, most of the indicators used in these studies have been aimed at assessing the use of interventions and at seeking out results based on pathology (i.e. postpartum hemorrhages, perineal tears, etc.). The aim of the MidconBirth study is to offer a new perspective on this issue and to contribute to research on the assessment of the quality of care that women receive when in labor. In most health care services, midwives are the primary providers of care throughout the process, but this is not always the case. The roles of different care providers vary depending on how health services and medical teams are organized. Most women who give birth are healthy or at least at low-risk during their pregnancies, and they tend to receive care from midwives who exercise complete autonomy from the onset of labor. The transfer of care during labor means that midwives are responsible for detecting any risk, problem or pathology requiring the intervention of another professional. In some cases, however, midwives' degree of autonomy is affected by the organizational structure of professional teams. In Spain, most women in labor receive care in obstetric units staffed by both obstetricians and midwives. These units have all the necessary technology to provide care for women regardless of the level of risk present in the pregnancy. Care is provided by professional teams organized into hierarchical structures. As a result, procedures are often determined by protocols, and professionals tend to work in accordance with a given center's organizational culture. In general, midwives tend to be responsible for women with low-risk pregnancies during labor. The degree of autonomy exercised by these midwives varies from center to center. Meanwhile, obstetricians act as consultants in these cases. In Ireland, intrapartum care for women with low-risk pregnancies is provided under the Mother and Infant Care Scheme [5], a program that promotes individualized care for low risk women in labor by midwives and under which obstetricians act only upon the request of these midwives.

This study examines the relationships between the intrapartum transfer of care from midwife to obstetrician-led maternity care and the obstetric unit size (OUS), intrapartum interventions and birth outcomes. 


\section{Methods}

This article is part of the MidconBirth study. This is a prospective, multicenter, cross-sectional study promoted by the COST Action IS1405 carried out in different hospitals in Europe. The protocol can be accessed through the registry ISRCTN14062994 [6]. For the purposes of this study, we selected births attended to in obstetric units in three different regions of Spain (Catalonia, Valencia and Castilla y León) representing hospitals with low volumes of births (<600 births per year) or Unit Size 1 (S1), medium (from 601 to 1200 births) and high annual volumes of births (1201 to 2400 births) or Unit Size (S2) and Unit Size (S3) respectively, as well as births attended to by a continuity of care team in Ireland (Cork), a hospital with high annual volume of births ( $>2400$ births) or Unit Size 4 (S4). Data were collected through an online platform in 2016-2019. The sample was limited to primiparous and multiparous women between 18 and 40 years of age with a singleton, cephalic presentation and uncomplicated pregnancy between 37 and 42 weeks of gestation. For this study, women with pregnancies classified as high or very high risk were excluded.

The reference population was of 5708 women. The sample size is calculated on the annual number of births of each participating center or midwife. To calculate the sample size ( $95 \%$ level of confidence) it is assumed an unknown proportion of births attended by midwives for each estimated population (50\%) in each setting, with a (+/-) $5 \%$ precision and a reposition proportion of $10 \%$. A minimum estimated sample size was 365 women to achieve a representative sample for each hospital in Spain and for the caseload midwifery team in Ireland. Data collection was conducted consecutively during the specified period until the minimum number of cases needed was reached. The primary outcome was transfer of care. The secondary outcomes included type of onset of labor, oxytocin stimulation, epidural analgesia, type of birth, episiotomy/perineal injury, postpartum hemorrhage, early initiation of breastfeeding and early skin-to-skin contact.

Descriptive statistics were used to summarize the women's characteristics. The statistical analysis was carried out using the SPSS program version 23.0 (IBM Corp. Released 2018. IBM SPSS Statistics for Windows, Version 25.0. Armonk, NY: IBM Corp.). Frequencies and percentages of the categories were calculated for all the variables. The standard deviation (SD) of the quantitative variables mean was calculated. The Chi-square test was used to analyze the statistical significance of the differences in the percentages of hospital groups between the variable categories; for risk factors for transfers of care, an odds ratio (OR) with a $95 \% \mathrm{Cl}$, was calculated. A multivariate logistic regression models were performed to ascertain the effects of studied co-variables on the likelihood that participants had a transfer of care. These models were adjusted using a stepwise variable selection process based on a likelihood ratio (LR). Nagelkerke's R2 was used to estimate the coefficient of determination from 0 to 1 . The significance level was set at $p<0.05$.

\section{Results}

The total sample analyzed was made up of 2126 cases. In terms of the regional distribution, Cork (Ireland) collected data on $7.1 \%$ (150) of the cases, $44.7 \%$ (951) were in Catalonia, 48.2\% (1025) in the regions of Valencia and Castilla y León (Spain). Regarding the obstetric unit size distribution, S1 represented $8.8 \%$ (187) of the cases, $51 \%$ (1086) were in S2, 33\% (703) were in S3 and 7.1\% (150) were in S4 (Figure 1).

The average age of the women in the study was $31.7 \pm 4.9$ years. Broken down by country of origin, $70.2 \%$ (1490) of the women were from Spain, $11.8 \%$ (250) were from elsewhere in Europe, 7.3\% (155) were from South or America, 8.1\% (172) were from Africa, 2.0\% (42) were from Asia, 0.5\% (10) were from the Middle East, 0,2\% (4) were from North America and for 0,1\% (3) of the women this data was missing. 36.3\% (772) of the women had attended university, $34.3 \%$ (729) had high school degrees, $25.2 \%$ (535) had only completed primary school, while for $4.2 \%$ (90) of the women the level of education was unknown or could not be classified. $52.3 \%(1111 / 2126)$ were primiparous, while the average gestation period before labor was $39.0 \pm 3.0$ weeks (range of $37-41)$.

The clinical characteristics of women in every US group are shown in Table 1, which also displays the statistically significant differences between US, with the exception of the use of epidural anesthesia $(p=0.632)$.

Of women who had dystocic births, in S1 we observed that $12.3 \%$ (23) of women required emergency caesarean sections; in S2 the percentage was $12.6 \%$ (137); in S3 16.5\% (116) and in S4 11.3\% (17). The differences here were statistically significant ( $\mathrm{p}<0.001)$. S1 displayed a lower percentage of transfer (29.4\%), fewer cases of labor stimulated with oxytocin (31.0\%) and fewer cases of induced labor (12.4\%). Meanwhile, S1 displayed the highest percentages of normal births (77.5\%), skin-to-skin contact between the mother and the newborn (96.3\%), and early initiation of breastfeeding (88.2\%).

S2 showed the highest percentage of induced labor (30.8\%), serious perineal injuries including episiotomies and third-and fourth-degree perineal tears (48.9\%), but this US showed the lowest percentage of postpartum hemorrhages (2.0\%).

S3 displayed the greatest percentage of transfer (54.9\%), of labor stimulation with oxytocin (70.4\%), of the use of epidural analgesics (85.5\%) and of dystocic births (37.1\%).

S4 showed the lowest proportion of births with epidural analgesia (18.7\%), was most likely to lack serious perineal injuries, characterized as cases where the perineum was intact or cases with second and third degree perineal tears (66.7\%), and had the highest percentage of the start of labor attended by midwives (99.3\%). However, this OUS also displayed the highest percentage of postpartum hemorrhages (7.3\%).

\section{Transfer analysis}

We were interested in analyzing the relationship between the transfer of care between the midwife and the obstetrician and the rest of the factors that influence a birth. For the purposes of this analysis, cases of elective cesarean sections (42) were excluded. Thus, the total number of cases analyzed was 
Midwives attended the start of the deliveries in $85.1 \%$ (1773/2084), and they attended during the expulsive phases of the deliveries in $59.4 \%$ (1237/2084) of cases. Meanwhile, obstetricians attended the start of the deliveries in $14.9 \%(311 / 2084)$ of cases, and they attended the end of deliveries in $40.6 \%$ $(847 / 2084)$ of cases.

In 55.5\% (1156/2084) of the deliveries, there was no TOC from the midwife to the obstetrician. In other words, in these cases midwife attended the whole labor and birth process. In terms of the distribution by US, the midwives in S1 were the least likely to transfer care (with 71.0\% [132/186] attending to the labor and birth in its entirety), followed by those in S4 (69.8\% [104/149]), S2 (56.0\% [1076/673]) and, finally, S3 (47.1\% [317/773]).

We conducted an analysis of the differences in the labor and birth processes and the associated perinatal results in each US, examining them in terms of whether or not there was a TOC during the process. For the variables analyzed, (type of start of labor, pharmacological stimulation of labor, use of epidural analgesics, type of birth and status of the perineum), statistically significant differences were found, both within each obstetric unit size and for the sample as a whole.

It is true that the midwife might not be directly responsible for the decision to induce labor, as this represents a departure from a normal birth because the onset is not spontaneous. However, this process is often determined by protocol and characterized by a shared responsibility of the midwife and the team of obstetricians [7].

Our analysis of the factors associated with a greater likelihood of transfer of care and the risks associated with this practice is displayed in the $2 \times 2$ tables and the odds ratio calculations (Table 2).

When there was no transfer of care, S4 recorded the highest proportion of spontaneous onset of labor [S4 93.3\% (97/104), compared with S1 at 90.9\% (120/132), S2 at 83.3\% (502/603), and S3 at 83.0\% (263/317)], and the differences found here were statistically significant (p<0.001). However, in S4 when labor is induced the likelihood of TOC is six times higher than when the onset of labor is spontaneous (OR=6.9; 95\% Cl: 2.5-18.6). S3 showed the highest rate of induced births attended by midwives in which no TOC occurred [17.0\% (54/317), while for S2 the figure was $16.7 \%$ (101/603), for S1 it was $9.1 \%$ $(12 / 132)$, and for S4 it was $6.7 \%(7 / 104)]$. The differences found were statistically significant $(p<0.001)$. S4 displayed the lowest frequency of oxytocin use in deliveries when no transfer of care occurred [13.5\% (14/104)]. Meanwhile, S3 registered the highest rate of pharmacological stimulation (55.5\% [176/317]). Additionally, in S4 TOC was more likely when oxytocin was used or labor was stimulated, in the latter case increasing by a factor of 29 with respect to when labor was not stimulated $(\mathrm{OR}=29.7,95 \% \mathrm{Cl}: 11.5-76.8 ; \mathrm{p}<0.001)$.

In cases where there was no TOC, S3 registered the lowest rate of use of epidural analgesics [75.1\% (238/317)], while S1 and S2 displayed the greatest tendency to administer them (78.8\% ([104/132] and 78.8\% [475/603], respectively). In S1, the risk of TOC was seven times higher when epidural analgesics were administered than when they were not (OR=7.0; $95 \% \mathrm{Cl}: 1.0-30.0 ; \mathrm{p}=0.003$, with the rate reaching $96.3 \%(52 / 54)$ in these cases. The lowest prevalence of TOC associated with the use of epidural analgesics was found in S2 (OR=2.8; 95\% Cl: 1.9-4.1; $p<0.001)$, where the figure was $91.3 \%$ (432/473).

In terms of the type of birth, all the spontaneous vaginal deliveries (SVD) in S4 (104/104) were attended by midwives, and therefore, there was no TOC. In contrast, none of the S4 births in which TOC occurred were SVD (0/45). Meanwhile, in S3, 35.1\% (125/356) of the deliveries that featured transfers of responsibilities were SVD and attended by obstetricians. The risk of TOC associated with labor ending in dystocia was the highest in S1 (S1 OR = 11.1, 95\% Cl: 6.6-18.6, p <0.001; S2 OR = 4.9, 95\% Cl: 4.3-5.6, p <0.001; S3 OR = 3.5, 95\% Cl:3.0-4.1, p <0.001).

With respect to the condition of the perineum when no TOC occurred, the S4 registered the highest number of cases with intact perineum or $1^{\text {st }}$ or $2^{\text {nd }}$ degree perineal tears [94.2\% (98/104)], compared with the figure of $72.3 \%(436 / 603)$ for S2. In contrast, when TOC occurred, the highest rate of intact perineum or $1^{\text {st }}$ or $2^{\text {nd }}$ degree perineal tears was found in S3 [44.9\% (160/356)], while S4 displayed the lowest rate [4.4\% (2/45)]. Meanwhile, these episiotomies or thirdor fourth-degree tears were present in $95.6 \%$ (43/45) of the cases in S4 when TOC was performed. The lowest rate in this regard was found in S3 [55.1\% (196/356]. Thus, when TOC occurs, the risk of episiotomy or third- or fourth-degree perineal tear (rather than an intact perineum or a first- or second-degree tear) was found in S4 to increase by a factor of $350(\mathrm{OR}=351.2,95 \% \mathrm{Cl}: 68.1-1810.3, \mathrm{p}<0.001)$. The risk of TOC and of episiotomy or third- and fourthdegree perineal tears was the lowest in S3 $(\mathrm{OR}=3.6,95 \% \mathrm{Cl}: 2.6-4.9, \mathrm{p}<0.001)$.

In addition, significant differences were found with regard to the presence of postpartum hemorrhages and early initiation of breastfeeding initiation only in the sample as a whole. This effect could be explained by the construction of a multivariate logistic regression model (Wald test) between these variables and the rest of the covariables studied, observing that the obstetric unit size, the induced onset of labor and having a episiotomy or grade III-IV injury were associated with an increasing risk of having postpartum hemorrhage. Women who had an episiotomy or a grade III-IV injury were twice as likely to have a postpartum hemorrhage compared with women who had an intact perineum or a I-Il degree tear [OR=2.5; CI95\%:1.4-4.4]; induction of labor is also a risk for postpartum hemorrhage [OR=1.8; Cl95\%:1.1-3.1]. Moreover, doing skin-to-skin was associated with an increased probability of early initiation of breastfeeding onset [OR=45.9; 95\% Cl: 28.89-72.77] (Table 3).

Furthermore, a multivariate logistic regression model was used in order to predict the variables that influenced the TOC. The related variables were OUS, parity, onset of labor, pharmacological stimulation of labor and episiotomy, with the US being the most influential variable (Table 4). Women in S3 have twice the probability [OR $=2.3 ; 95 \% \mathrm{Cl}: 1.4-3.6]$ of having a TOC compared to those in S4; being primiparous increases the probability of TOC by almost twice [OR $=1.9 ; 95 \% \mathrm{Cl}: 1.5-2.4]$; inducing labor rises this risk by almost three times [OR $=2.9 ; 95 \% \mathrm{Cl}$ : 2.3-3.8] in comparison with spontaneous onset of labor; the use of pharmacological stimulation and epidural analgesia are also risk factors for performing TOC $[\mathrm{OR}=1.3 ; 95 \% \mathrm{Cl}$ : 1.0-1.7, OR $=1.7 ; 95 \% \mathrm{Cl}$ : 
1.2-2.4, respectively] and performing an episiotomy increases the risk of TOC by five times [OR $=5.3 ; 95 \%$ Cl: 4.3-6.6]. The model obtained a percentage prediction of $73.4 \%$

\section{Discussion}

This cross-sectional study is part of a broader evaluation of maternity services in Spain and a center in Ireland. This paper focuses specifically on the intrapartum transfer of care of low-risk women between health care professionals in obstetric units (OU) and the associated clinical and organizational factors.

The majority of women whose labor care was initiated by a midwife remained in midwifery care throughout their labor and birth. However, there were statistical differences in the proportion of women transferred from midwifery care to obstetrician care according to the obstetric unit size (number of births). The hospitals with the lowest percentages of transfer of care were those in S1 and S4. These OUS groups had a transfer percentage of $29.4 \%$ and $31.2 \%$ respectively. Meanwhile, S3 had the highest transfer percentage (47.1\%). These transfer rates brought sharply into focus the differences between midwifery and medical models of care.

Out of all women, those in S4 (the highest number of births per year) were most likely to be provided one to one individualized care by a caseload midwifery team. These cases had the lowest frequency of oxytocin stimulation, epidural analgesia, episiotomy or severe perineal damage, and emergency caesarean section, and also registered among the lowest transfer percentages. Midwifery models recognize childbirth as a physiological process which has inherent sociocultural and psychological dimensions [8]. Our findings echo the strong existing evidence that suggests that continuity of care models achieve the best outcomes. For example, women who are attended in midwifery-led continuity models of care were found to be less likely to experience regional analgesia and severe perineal trauma $[4,9]$.

In general terms, the variables in this study showed some association with transfers of care, both within each US and in the sample as a whole. This was not the case, however, with postpartum hemorrhages, nor with early initiation of breastfeeding, for which associations were found for the sample as a whole but not for all the OUS. Logistic regression models show that for the sample as a whole, the covariables associated with postpartum hemorrhages were the US, the status of the perineum and the onset of labor, while the covariable associated with early initiation of breastfeeding was skin-to-skin contact between mother and infant[10]. Thus, we could conclude that these act as confounding variables for the sample as a whole.

We observed that certain factors connected to poor perinatal outcomes, such as the induction of labor, the stimulation of labor with oxytocin and the use of epidural analgesics; also tend to be associated with the transfer of care from the midwife to the obstetrician. This in turns leads outcomes such as dystocic birth and episiotomies or third- or fourth-degree tears. This could be explained by the high prevalence of obstetric interventions, especially in low-risk women, which trigger the need of performing a cascade of subsequent childbirth interventions [11].

We also confirmed that there is a high degree of variability in the intrapartum care given to low-risk women, as well as a high rate of interventionism in all the hospitals, results that echo the findings of other studies [12-15]. Among the cohort of women planning to give birth in Spanish obstetric units, we found considerably greater variation in intervention percentages than we would expect, and this is not explained by any known differences in maternal characteristics. Our findings show a significant association between the size of the unit, the professional that initiates the intrapartum care and the number of obstetric interventions performed; the smaller the OU, the more likely women are to receive care from a midwife and the less likely they are to receive obstetric interventions. Our findings confirm that variations in intervention rates are not fully explained clinical characteristics of women planning to give birth or women's preferences. Since low-risk women have different outcomes according to the hospital where they give birth, this may reflect a more interventional practice style and woman-centered care might not be implemented or interpreted in the same way at all places [16, 17].

The OUS was also the variable that appeared to be most influential for women to have a TOC above parity, onset of labor, pharmacological stimulation of labor, and episiotomy. Although the predictive model is acceptable in terms of TOC prediction, the variability explained is estimated only at $37.9 \%$. Thus, this result must be interpreted with caution. It seems reasonable that every OUS may have a different organization of care model in which the rest of the variables involved are adapted according to the type of intervention in childbirth. Nevertheless, all obstetric units should include a philosophy of supporting normal birth and women centered care[18][18].

On the one hand, the care in S1 (units with smallest number of births)and in S4 (caseload team in Ireland) was initiated in almost in all the cases by a midwife, and these cases had the lowest transfer rate out of all women in both countries and the highest spontaneous vaginal birth rate. On the other hand, larger OUs (S3) had the highest prevalence of a high level of oxytocin stimulation, epidural use, and instrumental births, and in these cases the majority of women were transferred to an obstetrician. These findings are in agreement with previous research that suggests that "low-risk" women are more likely to have a spontaneous vaginal birth in hospitals with smaller maternity departments or in midwifery-led units that operate a policy geared towards normal birth $[17,19,20]$. However, the findings differ with earlier studies that had showed mixed or inconclusive results on the relationship between unit size and intervention rates[21].

In general, the institutional factors affecting intervention rates are poorly understood. However, it is critical to come to an understanding of childbirth practices as an organizational cultural phenomenon. The culture of a given work environment may encourage care providers to take similar decisions, and variations are therefore not merely individual. Differences in perceptions and attitudes may result in differences in local practice and guidelines[22].

In Spain, midwives' scope of practice follows European directives[7], and the Ministry of Health promotes assistance based on the best evidence available, with appropriate use of technology for avoiding unnecessary procedures[23]. Intrapartum care is performed in hospitals staffed by teams of midwives and obstetricians. However, there is a significant lack of midwifery staffing in the country[24]. In fact, in the region of Catalonia, all hospitals have more

Page 5/12 
obstetricians than midwives, with just one exception: public hospitals classified as S3 [25], which nonetheless showed the highest proportion of transfers in our study. Furthermore, we found a strong link between larger hospitals and higher numbers of spontaneous vaginal deliveries attended by obstetricians when women were transferred in Spain. Actually, there were no reported cases of women having a spontaneous vaginal birth assisted by an obstetrician in Ireland. This might also reflect the lack of midwifery staffing in Spain and a lack of autonomy in midwives' practice in the country, especially in S3 hospitals. Transfers might be associated with the embedded social and cultural norms of an institution or their consequences on birth practices[25, 26].

We would like to highlight that in S2 and S3 the percentages of induction of labor, oxytocin stimulation, severe perineal damage and episiotomy are considerably higher than in many European countries[27]. It is then plausible to assume that the elevated number of obstetric interventions and transfers of care is at least partly attributable to the medical obstetric practice that is predominant in Spain, which tends to be highly interventionist[25]. Unwarranted variation in medical care is also associated with non-evidence-based practice[16, 28]. In addition, recent studies have shown some association between junior doctor staffing levels and intervention rates[29,30]. This could be interpreted as negative, as the majority of obstetricians and midwives are trained in these units.

These results should spark debate on the effect the organization of services may have in the Spanish context. All the public maternity units are technically orientated and able to attend both high risk and low-risk births. This raises issues as to the dubious benefits of giving birth in mixed environments, as recent studies have shown positive health results when healthy women are treated in midwife-led units that are separated from conventional obstetric units [4, 31].

Previous research has shown that a given hospital's policies and procedures, inadequate staffing, technology-focused care, and a lack of continuity of care are all barriers to a more humanized approach to birth at specialized hospitals[32]. The findings here, therefore, suggest that the current model of maternity care in these Spanish hospitals should be reconsidered in light of the impact these practices may have on maternal and neonatal outcomes. In addition, expanding midwife-led maternity services for eligible women may offer a means of reducing costs compared to the current leading model of care [33, 34].

This is the first study that has examined transfer of care and the associated clinical and organizational factors in Spain. A strength of this study is that we were able to evaluate the transfer of care in a homogeneous cohort of "low-risk" women that received care in different settings and health care systems in Spain and Ireland. The limitation of the existing socio-cultural studies of birth practices is that they fail to explore the organizational culture dimensions of the institutions and their role and power to bring about changes to humanize birth practices. Nonetheless, this study has limitations of its own which should be noted. The first concerns the study's observational design. It is not possible to establish causal relationships in our study, for instance, between transfer of care and type of birth. Whether there is a causal relationship between these variables needs to be investigated in further research. However, the results are consistent with the findings from previous studies that showed better maternal outcomes when midwifery continuity models of care are implemented[4]. Secondly, another limitation, and one that probably applies to all research in this area, is the extremely limited available data on the characteristics of maternity units in Spain. The data we gathered on the characteristics of the included obstetric units in the study included their size and the model of care offered. However, we were unable to consider whether the women could have opted for an alternative place of birth or to look at the actual reason for transfer of care or the level of midwifery staffing[32], and we acknowledge this could have provided us with a more accurate interpretation of the results. These findings should therefore be interpreted with caution.

As stated in a Lancet series on midwifery, the available data strongly suggest an urgent need for more research to assess the appropriate interventions for childbirth[1]. Qualitative research is required to improve our understanding of the barriers and facilitating factors midwives encounter when promoting normal birth in specialized hospitals, where highly technological and medicalized birth practices exist. Our research group is currently working on identifying these in obstetric units in Catalonia, Spain. In addition, it is very important to gather the opinions of service users and professionals if we aim to provide woman-centered care[35].

Midwifery-led care should be promoted in Spain to support midwives and allow them to work autonomously to their full scope of practice[7]. Developing a national strategy taking into consideration women's wishes and demands and the latest evidence for maternity care could be a potential approach[5]. Additionally, in-house professional development programs, including for medical and midwifery staff, are necessary to address the lack of knowledge regarding the concept of midwifery autonomy. Active involvement by midwives in groups drafting hospital guidelines and in-service development programs may also contribute to their professional development[36, 37]. Finally, following national guidelines and applying recommendations of the WHO might help hospitals in Spain reduce in their use of interventions for women who have a spontaneous onset of labor and minimize unwarranted variation in the use of interventions[22, 32, 35].

\section{Conclusions}

This exploratory study of "low-risk" births planned in obstetric units suggests that the size of the obstetric unit and the level of provision of midwifery-led care within the institution may explain some of the variation in intervention rates. To provide maternity care of optimal quality, public health stakeholders need to be aware of the childbirth practices in different organizations and then ensure that these conform to women's and their families' needs.

\section{List Of Abbreviations}

LR: Likelihood Ratio

OR: Odds Ratio

OU: Obstetric Unit 
OUS: Obstetric Unit Size

SVD: Spontaneous Vaginal Delivery

S1: Obstetric unit size 1 ( $<600$ births per year)

S2: Obstetric unit size 2 (from 601 to 1200 births per year)

S3: Obstetric unit size 3 (1201 to 2400 births per year)

S4: Obstetric unit size 4 (>2400 births per year)

TOC: Transfer of Care

\section{Declarations}

\section{Ethics approval and consent to participate}

The MidconBirth study was approved by the ethics committee of the coordinating center (Clinical Research Ethics Committee of Parc Salut Mar 2016/6785/I) ISRCTN registry 17833269 and later by the ethics committee of each participating center (Clinical Research Ethics Committee of the Catalan Hospitals Union Foundation (CPMP/ICH/135/95), Clinical Research Ethics Committee of Rio Ortega Hospital (117/16), Clinical Research Ethics Committee (CREC) Cork Ref ECM4 (y 09/05/17 ECM3qqqqq), Human Ethics Committee at Hospital Universitario de La Ribera Research Ethics Committee and Research Commission and the Spanish Medicines and Medical Devices Agency approved the study (HULR15/12-01), Research Ethics Committee of Complejo Asistencial Universitario de Palencia (CIB-2017005) and Research Ethics Committee of Hospital Verge dels Lliris. Ethics committee approval was required for each participating hospital. Since this is an observational study in which data was anonymized, no consent was required from the women cared for in the participating centers. If a hospital required consent from the women under their care, written consent was obtained. Further information and documentation are available on request.

\section{Consent for publication}

Not applicable.

\section{Availability of data and material}

The original dataset is available from the corresponding author upon reasonable request.

\section{Competing interests}

The authors declare that they have no competing interests.

\section{Funding}

The MidconBirth Study was funded by the Catalan Council of Nurses - Midwives Commission. This contributed to the creation of a web-based platform for data collection. This project has also been funded by the Conselleria de Educación, Investigación, Cultura y Deporte of the Generalitat Valenciana in its call for grants for the conduction of R\&D\&l projects developed by emerging research groups in 2018 (Reference GV/2018/036). This grant contributed to the dissemination of the study results. And finally, this study contributes to COST action IS 1405.

\section{Authors' contributions}

AM, RVC, ROC and RE were involved in conceptualizing the study. AMA and RE designed the protocol and led the project. AM, RVC and RE conducted the statistical analysis. All authors contributed to the interpretation of the results. AMA, RE and RVC wrote the first draft of the manuscript and all authors read and approved the final manuscript. RE is the principal investigator for the MidconBirth Study.

\section{Acknowledgements}

We are grateful to all women and their families who participated in this study and all midwives who collected data for the MidconBirth study and thank them for their support with this research.

\section{References}

1. Miller S, Abalos E, Chamillard M, Ciapponi A, Colaci D, Comandé D, et al. Beyond too little, too late and too much, too soon: a pathway towards evidencebased, respectful maternity care worldwide. 2016;6736:1-17.

2. World Health Organization. WHO recommendations: intrapartum care for a positive childbirth experience. Geneva: World Health Organization; 2018. Available at: https://www.who.int/reproductivehealth/publications/intrapartum-care-guidelines/en/

3. Marshall JL, Spiby H, McCormick F. Evaluating the 'Focus on Normal Birth and Reducing Caesarean section Rates Rapid Improvement Programme': A mixed method study in England. Midwifery. 2015;31:332-40. 
4. Sandall J, Soltani H, Gates S, Shennan A, Devane D. Midwife-led continuity models versus other models of care for childbearing women. Cochrane Database Syst Rev. 2016;(4):CD004667.

5. Department of Health. Creating a Better Future Together National Maternity Strategy 2016-2026. Dublin: Department of Health; 2016. Available at: https://health.gov.ie/wp-content/uploads/2016/01/Final-version-27.01.16.pdf

6. Escuriet R, García-Lausin L, Salgado-Poveda I, Casañas R, Robleda G, Canet O, et al. Midwives' contribution to normal childbirth care. Cross-sectional study in public health settings. MidconBirth Study protocol. Eur J Midwifery. 2017;1 September:1-5.

7. European Comission. European Parliament and the Council Directive 2005/36/EC: The professional qualifications directive (Directive 2013/36/EC). Brussels: Off J Eur Union. 2013.

8. Walsh D, Newburn M. Towards a social model of childbirth: part two. Br J Midwifery. 2002;10:540-4.

9. Hodnett ED. Continuity of caregivers for care during pregnancy and childbirth. Cochrane Database Syst Rev. 2008, (4): CD000062.

10. Vila-Candel R, Duke K, Soriano-Vidal FJ, Castro-Sánchez E. Affect of early skin-to-skin mother-infant contact in the maintenance of exclusive Breastfeeding: Experience in a health department in Spain. J Hum Lact. 2018;34:304-12.

11. Petersen A, Poetter U, Michelsen C, Gross MM. The sequence of intrapartum interventions: A descriptive approach to the cascade of interventions. Arch Gynecol Obstet. 2013;288:245-54.

12. Nippita TA, Lee YY, Patterson JA, Ford JB, Morris JM, Nicholl MC, et al. Variation in hospital caesarean section rates and obstetric outcomes among nulliparae at term: A population-based cohort study. BJOG An Int J Obstet Gynaecol. 2015;122:702-11.

13. Glantz JC. Obstetric Variation, intervention, and outcomes: Doing more but accomplishing less. Birth. 2012;39:286-90.

14. Lutomski JE, Morrison JJ, Lydon-Rochelle MT. Regional variation in obstetrical intervention for hospital birth in the Republic of Ireland, $2005-2009$. BMC Pregnancy Childbirth. 2012;12:123.

15. Plough AC, Galvin G, Li Z, Lipsitz SR, Alidina S, Henrich NJ, et al. Relationship between labor and delivery unit management practices and maternal outcomes. Obstet Gynecol. 2017;130(2):358-65.

16. Panda S, Begley C, Daly D. Clinicians' views of factors influencing decision-making for caesarean section: A systematic review and metasynthesis of qualitative, quantitative and mixed methods studies. PLoS One. 2018;13:1-27.

17. Behruzi R, Hatem M, Goulet L, Fraser W, Misago C. Understanding childbirth practices as an organizational cultural phenomenon: A conceptual framework. BMC Pregnancy Childbirth. 2013;13:205.

18. Kennedy HP, Grant J, Walton C, Shaw-Battista J, Sandall J. Normalizing birth in England: A qualitative study. J Midwifery Women's Heal. 2010;55:2629.

19. Schulkind L, Shapiro TM. What a difference a day makes: Quantifying the effects of birth timing manipulation on infant health. J Health Econ. 2014;33:139-58.

20. Biro MA, Knight M, Wallace E, Papacostas K, East C. Is place of birth associated with mode of birth? the effect of hospital on caesarean section rates in a public metropolitan health service. Aust New Zeal J Obstet Gynaecol. 2014;54:64-70.

21. Coulm B, Le Ray C, Lelong N, Drewniak N, Zeitlin J, Blondel B. Obstetric Interventions for low-risk pregnant women in France: Do maternity unit characteristics make a difference? 2012;39:183-91.

22. De Jong JD, Groenewegen PP, Westert GP. Mutual influences of general practitioners in partnerships. Soc Sci Med. 2003;57:1515-24.

23. Ministry of Health and Consumers' affairs Spain. Strategy for Assistance at Normal Childbirth in the National Health System. Madrid: Ministry of Health and Consumers' affairs; 2008. Available at: https://www.mscbs.gob.es/organizacion/sns/planCalidadSNS/pdf/InformeFinalEAPN_revision8marzo2015.pdf

24. Federación de Asociación de Matronas de España. Informe de situación se las matronas en España. Madrid: Federación de Asociación de Matronas de España; 2014. Available at: https://federacion-matronas.org/wp-content/uploads/2018/01/informe-situacion-matronas-espana2014-mod3.pdf

25. Escuriet-Peiró R, Goberna-Tricas J, Pueyo-Sanchez MJ, Garriga-Comas N, Úbeda-Bonet I, Caja-López C, et al. Impact of maternity care policy in Catalonia: A retrospective cross-sectional study of service delivery in public and private hospitals. BMC Pregnancy Childbirth. 2015;15:1-10.

26. Escuriet R, Pueyo M, Biescas H, Colls C, Espiga I, White J, et al. Obstetric interventions in two groups of hospitals in Catalonia: a cross-sectional study. BMC Pregnancy Childbirth. 2014;14:143.

27. EURO-PERISTAT project. European Perinatal Health Report: the health and care of pregnant women and babies in Europe in 2010. 2013. Available at:. 2010. http://www.europeristat.com/images/doc/Peristat 2013 V2.pdf

28. Goodman DC. Unwarranted Variation in Pediatric Medical Care. Pediatr Clin North Am. 2009;56:745-55.

29. Joyce R, Webb R, Peacock J. Predictors of obstetric intervention rates: Case-mix, staffing levels and organisational factors of hospital of birth. J Obstet Gynaecol. 2002;22:618-25.

30. Coonrod DV, Drachman D, Hobson P, Manriquez M. Nulliparous term singleton vertex cesarean delivery rates: institutional and individual level predictors. Am J Obstet Gynecol. 2008;198:694.e1-e11.

31. Rowe RE, Townend J, Brocklehurst P, Knight M, Macfarlane A, McCourt C, et al. Service configuration, unit characteristics and variation in intervention rates in a national sample of obstetric units in England: An exploratory analysis. BMJ Open. 2014;4:1-9.

32. Campbell P, Rudisill P. Psychosocial needs of the critically ill obstetric patient: the nurse's role. Crit Care Nurs Q 2006, 29(1):77-80. 
33. Tracy SK, Hartz DL, Tracy MB, Allen J, Forti A, Hall B, et al. Caseload midwifery care versus standard maternity care for women of any risk: M@NGO, a randomised controlled trial. Lancet. 2013;382:1723-32.

34. Ryan P, Revill P, Devane D, Normand C. An assessment of the cost-effectiveness of midwife-led care in the United Kingdom. Midwifery. 2013;29:368-76.

35. Greenhalgh T, Humphrey C, Hughes J, MacFarlane F, Butler C, Pawson R. How Do You Modernize a Health Service? A Realist Evaluation of Whole-Scale Transformation in London. Milbank Q. 2009;87:391-416.

36. Herron A. "Autonomy and midwifery" [dissertation]. London: Middlesex University, 2009;2-6.

37. International Confederation of Midwives. Strategy 2017- 2020. The Hague: International Confederation of Midwives; 2017. Available at: https://www.internationalmidwives.org/assets/files/general-files/2018/04/final-copy-icm-strategy-\%202017-20-online.pdf

\section{Tables}

Table 1. Characteristics of the sample, by obstetric unit size $(n=2126)$.

\begin{tabular}{|c|c|c|c|c|c|c|c|c|c|c|}
\hline & \multicolumn{9}{|c|}{ Obstetric Unit Size } & \multirow[b]{4}{*}{ p* } \\
\hline & \multicolumn{2}{|c|}{ S1 } & \multicolumn{2}{|c|}{$\mathrm{S} 2$} & \multicolumn{2}{|c|}{ S3 } & \multicolumn{2}{|c|}{$\mathrm{S} 4$} & \multirow[b]{3}{*}{ Total } & \\
\hline & \multicolumn{2}{|c|}{$\mathrm{n}=187$} & \multicolumn{2}{|c|}{$\mathrm{n}=1086$} & \multicolumn{2}{|c|}{$\mathrm{n}=703$} & \multicolumn{2}{|c|}{$\mathrm{n}=150$} & & \\
\hline & $\mathrm{n}$ & $\%$ & $\mathrm{n}$ & $\%$ & $\mathrm{n}$ & $\%$ & $\mathrm{n}$ & $\%$ & & \\
\hline Onset of labor & & & & & & & & & & \multirow[b]{4}{*}{$<0.001$} \\
\hline Spontaneous & 163 & 87.2 & 745 & 68.6 & 492 & 70.0 & 127 & 84.6 & 1527 & \\
\hline Induced & 23 & 12.3 & 331 & 30.5 & 181 & 25.7 & 22 & 14.7 & 557 & \\
\hline C-section & 1 & 0.5 & 10 & 0.9 & 30 & 4.3 & 1 & 0.7 & 42 & \\
\hline \multicolumn{11}{|l|}{ Pharmacological stimulation of labor } \\
\hline No & 129 & 69.0 & 419 & 38.6 & 208 & 29.6 & 99 & 66.0 & 855 & \\
\hline Yes & 58 & 31.0 & 667 & 61.4 & 495 & 70.4 & 51 & 34.0 & 1271 & $<0.001$ \\
\hline \multicolumn{11}{|l|}{ Epidural analgesia } \\
\hline No & 30 & 16.0 & 169 & 15.6 & 102 & 14.5 & 28 & 18.7 & 329 & \\
\hline Yes & 157 & 84.0 & 917 & 84.4 & 601 & 85.5 & 122 & 81.3 & 1797 & 0.632 \\
\hline \multicolumn{11}{|l|}{ Type of birth } \\
\hline Normal & 145 & 77.5 & 757 & 69.7 & 442 & 62.9 & 104 & 69.3 & 1448 & \\
\hline Dystocic & 42 & 22.5 & 329 & 30.3 & 261 & 37.1 & 46 & 30.7 & 678 & $<0.001$ \\
\hline \multicolumn{11}{|l|}{ Weight of newborn (g) } \\
\hline$<2500$ & 5 & 2.7 & 15 & 1.4 & 6 & 0.9 & 0 & 0.0 & 26 & \\
\hline $2501-3000$ & 34 & 18.2 & 227 & 20.9 & 142 & 20.2 & 10 & 6.7 & 413 & \\
\hline $3001-3500$ & 84 & 44.9 & 505 & 46.5 & 302 & 43.0 & 52 & 34.7 & 943 & \\
\hline $3501-4000$ & 49 & 26.2 & 284 & 26.2 & 212 & 30.2 & 53 & 35.3 & 598 & \\
\hline$>4001$ & 15 & 8.0 & 55 & 5.1 & 41 & 5.8 & 35 & 23.3 & 146 & $<0.001$ \\
\hline \multicolumn{11}{|l|}{ Perineum } \\
\hline No episiotomy or 1st or 2nd Degree & 111 & 59.4 & 555 & 51.1 & 398 & 56.6 & 100 & 66.7 & 1164 & \\
\hline Presence of episiotomy or 3rd or 4th Degree & 76 & 40.6 & 531 & 48.9 & 305 & 43.4 & 50 & 33.3 & 962 & 0.001 \\
\hline \multicolumn{11}{|l|}{ Postpartum hemorrhage } \\
\hline No & 182 & 97.3 & 1064 & 98.0 & 676 & 96.2 & 139 & 92.7 & 2061 & \\
\hline Yes & 5 & 2.7 & 22 & 2.0 & 27 & 3.8 & 11 & 7.3 & 65 & 0.002 \\
\hline \multicolumn{11}{|l|}{ Early skin-to-skin contact } \\
\hline Yes & 180 & 96.3 & 959 & 88.3 & 646 & 91.9 & 141 & 94.0 & 1926 & \\
\hline No & 7 & 3.7 & 127 & 11.7 & 57 & 8.1 & 9 & 6.0 & 200 & 0.001 \\
\hline \multicolumn{11}{|l|}{ Early initiation of breastfeeding } \\
\hline Yes & 165 & 88.2 & 823 & 75.8 & 552 & 78.6 & 124 & 83.2 & 1664 & \\
\hline No & 22 & 11.8 & 263 & 24.2 & 150 & 21.4 & 25 & 16.8 & 460 & 0.001 \\
\hline \multicolumn{11}{|l|}{ Professional attending the onset of labor } \\
\hline Midwife & 181 & 96.8 & 901 & 83.0 & 546 & 77.7 & 149 & 99.3 & 1777 & \\
\hline Obstetrician & 6 & 3.2 & 185 & 17.0 & 157 & 22.3 & 1 & 0.7 & 349 & $<0.001$ \\
\hline Professional attending the birth & & & & & & & & & & \\
\hline Midwife & 132 & 70.6 & 677 & 62.3 & 324 & 46.1 & 104 & 69.3 & 1237 & \\
\hline Obstetrician & 55 & 29.4 & 409 & 37.7 & 379 & 53.9 & 46 & 30.7 & 889 & $<0.001$ \\
\hline Birth attended by midwife from start to end & & & & & & & & & & \\
\hline Yes & 132 & 70.6 & 603 & 55.5 & 317 & 45.1 & 104 & 69.3 & 1156 & \\
\hline & & & & & & & & & 970 & \\
\hline No & 55 & 29.4 & 483 & 44.5 & 386 & 54.9 & 46 & 30.7 & & $<0.001$ \\
\hline
\end{tabular}

Table 2. Transfer of care distribution between different studied variables, and Odds Ratio and $95 \%$ confidence intervals ( $N=2084)$. 
Obstetric Unit Size

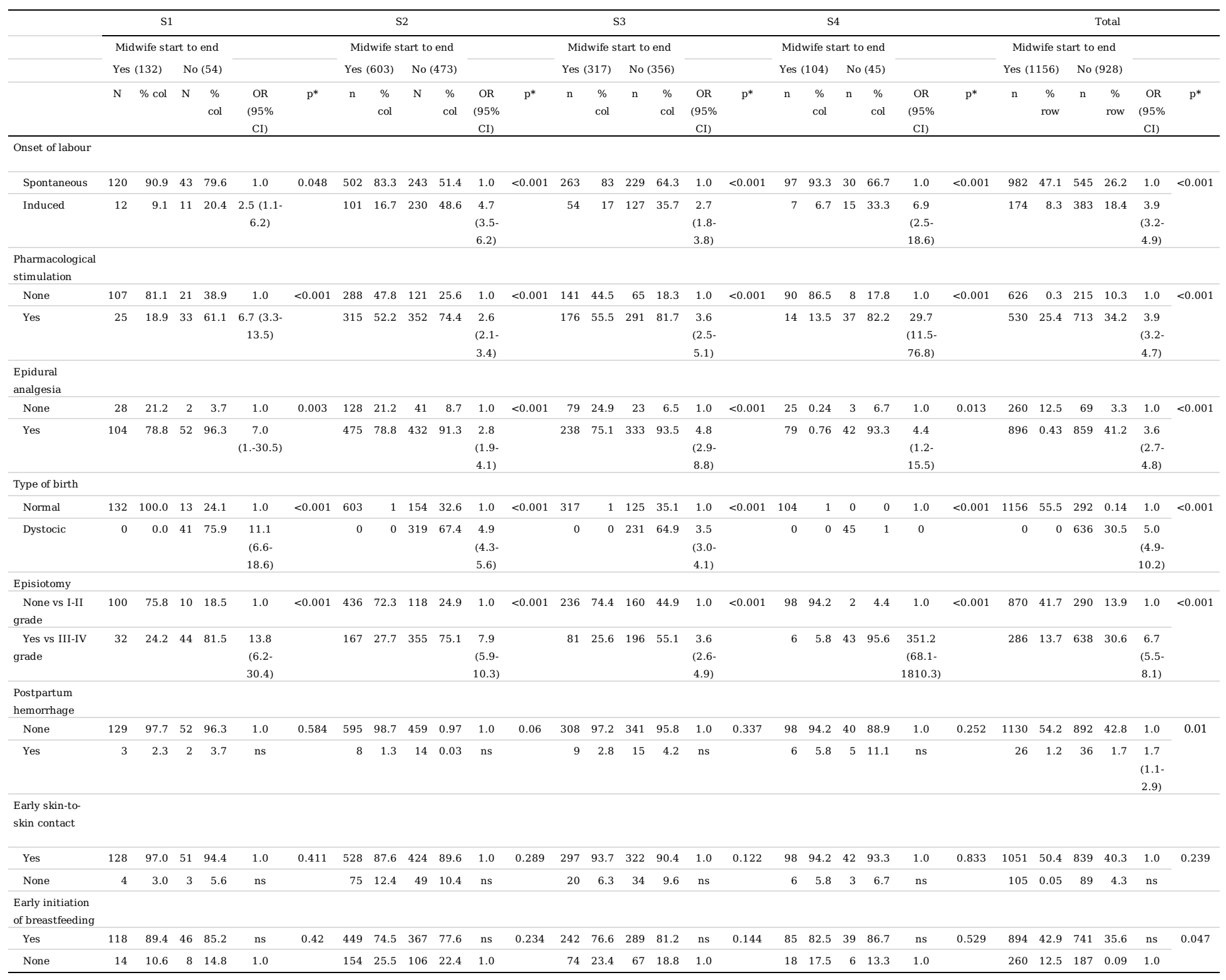

\section{p*: Chi-squared test}

Abbreviation: CI, Confidence Interval; ns, non-significant value $>0.05$

Crude odds ratio calculated using the risk of the contingency tables (2x2) between the transfer and the studied variables.

Table 3. Multivariate logistic regression between hemorrhage, breastfeeding and related covariables $(\mathrm{N}=2084)$.

\begin{tabular}{llrrrr}
\hline & & \multicolumn{3}{c}{ CI 95\% for EXP (B) } \\
& & p-value & OR & Lower & Upper \\
\hline Hemorrhage & S4 (Ref) & 0.001 & & & \\
& S1 & 0.045 & 0.3 & 0.1 & 0.9 \\
\cline { 2 - 6 } & S2 & 0.000 & 0.2 & 0.9 & 0.4 \\
\cline { 2 - 6 } & S3 & 0.014 & 0.4 & 0.9 & 0.8 \\
\cline { 2 - 6 } & Induced & 0.028 & 1.8 & 1.1 & 3.1 \\
\cline { 2 - 6 } & Episiotomy & 0.001 & 2.6 & 1.5 & 4.4 \\
\cline { 2 - 6 } & Constant & 0,001 & 0.1 & & \\
\hline Breastfeeding & Skin-to-skin contact & 0.001 & 45.9 & 28.9 & 72.8 \\
\cline { 2 - 6 } & Constant & 0.001 & 0.2 & & \\
\hline
\end{tabular}

Table 4. Multivariate logistic regression of variables related to TOC $(\mathrm{N}=2084)$ 


\begin{tabular}{|c|c|c|c|c|c|}
\hline & & \multirow[t]{2}{*}{ p-value } & \multirow[t]{2}{*}{ OR } & \multicolumn{2}{|c|}{$95 \%$ CI OR } \\
\hline & & & & Lower & Upper \\
\hline \multicolumn{6}{|l|}{ Unit Size } \\
\hline & S4 (Ref) & 0.001 & & & \\
\hline & $\mathrm{S} 1$ & 0.498 & 0.8 & 0.5 & 1.4 \\
\hline & $\mathrm{S} 2$ & 0.273 & 1.3 & 0.8 & 2.0 \\
\hline & S3 & 0.001 & 2.3 & 1.5 & 3.6 \\
\hline \multicolumn{6}{|l|}{ Parity } \\
\hline \multicolumn{6}{|c|}{ Multiparous (Ref) } \\
\hline & Nulliparous & 0.001 & 2 & 1.6 & 2.4 \\
\hline \multicolumn{6}{|c|}{ Beginning of delivery } \\
\hline \multicolumn{6}{|c|}{ Spontaneous (Ref) } \\
\hline & Induction & 0.001 & 3 & 2.3 & 3.8 \\
\hline \multicolumn{6}{|c|}{ Pharmacologic stimulation } \\
\hline \multicolumn{6}{|c|}{ None (Ref) } \\
\hline & Yes & 0.011 & 1.4 & 1.1 & 1.8 \\
\hline \multicolumn{6}{|c|}{ Epidural analgesia } \\
\hline \multicolumn{6}{|c|}{ None (Ref) } \\
\hline & Yes & 0.001 & 1.7 & 1.2 & 2.4 \\
\hline \multicolumn{6}{|c|}{ Episiotomy } \\
\hline \multicolumn{6}{|c|}{ None (Ref) } \\
\hline & Yes & 0.001 & 5.3 & 4.3 & 6.6 \\
\hline Constant & & 0.001 & 0.1 & & \\
\hline
\end{tabular}

Nagelkerke $\mathrm{R}^{2}=0.379$; percentage prediction $=73.4 \%$

\section{Figures}




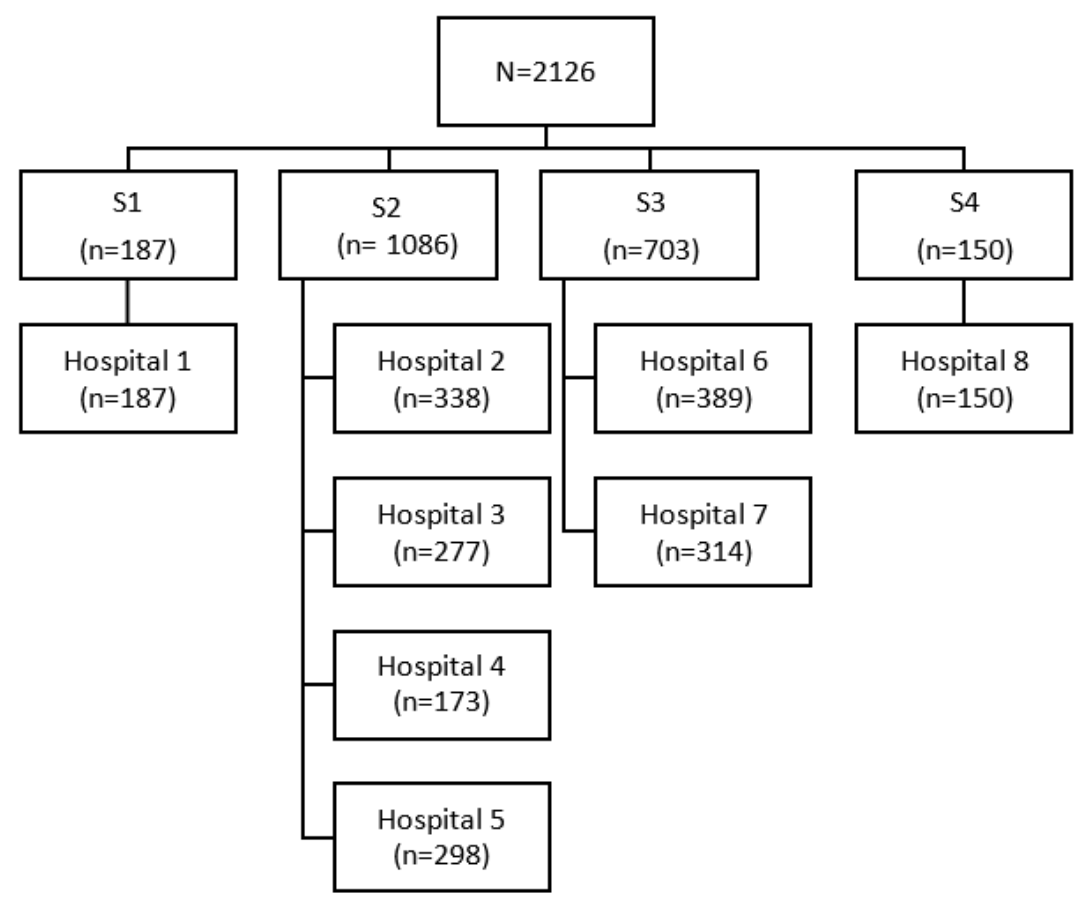

Figure 1

Study flow chart. 